\title{
IRREDUCIBLE LOCALLY NILPOTENT FINITARY SKEW LINEAR GROUPS
}

\author{
by B. A. F. WEHRFRITZ \\ (Received 15th February 1993)
}

\begin{abstract}
Let $V$ be a left vector space over the arbitrary division ring $D$ and $G$ a locally nilpotent group of finitary automorphisms of $V$ (automorphisms $g$ of $V$ such that $\operatorname{dim}_{D} V(g-1)<\infty$ ) such that $V$ is irreducible as $D-G$ bimodule. If $\boldsymbol{V}$ is infinite dimensional we show that such groups are very rare, much rarer than in the finitedimensional case. For example we show that if $\operatorname{dim}_{D} V$ is infinite then $\operatorname{dim}_{D} V=|G|=\aleph_{0}$ and $G$ is a locally finite $q$-group for some prime $q \neq \operatorname{char} D$. Moreover $G$ is isomorphic to a finitary linear group over a field. Examples show that infinite-dimensional such groups $G$ do exist. Note also that there exist examples of finitedimensional such groups $G$ that are not isomorphic to any finitary linear group over a field. Generally the finite-dimensional examples are more varied.
\end{abstract}

1991 Mathematics subject classification: 20H99, $20 \mathrm{~F} 19$.

\section{Introduction}

Throughout this paper $D$ denotes a division ring, $F$ some central subfield of $D$ and $V$ a left vector space over $D$. The finitary general linear group $F G L(V)$ or $F$ Aut $_{D} V$ over $V$ is the subgroup of $A_{u t} V$ of $D$-automorphisms $g$ of $V$ such that $[V, g]=V(g-1)$ has finite (left) dimension over $D$. By a finitary skew linear group we mean any subgroup $G$ of $F G L(V)$ for any $D$ and $V$. In [12] we showed that irreducible locally nilpotent subgroups of $F G L(V)$ for $V$ infinite-dimensional over $D$, for $D$ any division ring, are rather special, especially if the group is also torsion-free. Here we follow up this theme by showing that these finitary skew linear groups are really very rare indeed. (In [13] we also considered these groups, but only for certain restricted division rings.)

Theorem. Let $G$ be a locally nilpotent, honogeneous (e.g. irreducible) subgroup of $F G L(V)$, where $V$ has infinite dimension over $D$. Then $\operatorname{dim}_{D} V$ is countable, $G$ is $a$ countably infinite, locally finite q-group for some prime $q \neq$ char $D, G$ is isomorphic to a completely reducible monomial finitary linear group over any algebraically closed field of characteristic not $q$ and $G$ is isomorphic to a subgroup of the restricted permutational wreath product $\left.G L(W)\right|_{\Omega} S$, for some finite-dimensional $D$-space $W$, countably infinite set $\Omega$ and transitive locally finite q-subgroup $S$ of $F \operatorname{Sym}(\Omega)$.

Corollary. Let $G$ be a locally nilpotent, completely reducible subgroup FGL(V). Then each finitely generated subgroup of $G$ is also completely reducible. 
Is every subgroup $X$ of the group $G$ in the Corollary completely reducible? Note that the $F$-subalgebra of End $_{D} V$ generated by such an $X$ is semiprime by $([12,2.7 \mathrm{~d}$ and $2.9 \mathrm{~b}]$ ), or see it directly. There do exist locally nilpotent (even abelian) skew linear groups that are not completely reducible, but all of whose finitely generated subgroups are completely reducible, see $[7,1.1 .8]$. Such groups necessarily generate semiprime algebras over the centres of the ground division rings. The question is, can one of these groups lie in a locally nilpotent completely reducible skew linear group?

Apart from the proof of the above theorem we give a number of examples. First we show that groups $G$ as in the Theorem do exist; that is, we give examples of irreducible, locally nilpotent finitary skew linear groups of infinite dimension. Such groups are periodic (by the Theorem) and cannot be nilpotent or even hypercentral, indeed they have zero central height. It follows from the Theorem that an irreducible torsion-free locally nilpotent finitary skew linear group $G$ must be finite-dimensional. If also $D$ is a field (more generally if $D$ is either locally finite-dimensional as a ring or special in the sense of $[13]$, see $([13,1.3 \mathrm{~d}$ and $1.4 \mathrm{~d}])$ then $G$ is even abelian. Thus secondly we give examples of irreducible torsion-free nilpotent skew linear groups of arbitrary positive dimension and arbitrary positive nilpotency class.

Finally we give examples to show that the Suprunenko-Tyskevic Theorem $([8,7.15])$ does not extend from linear to skew linear groups in general (see the preamble to 17, below for the details). This arises during the proof of the theorem above, since if the Suprunenko-Tyskevic Theorem did extend it would have been useful at one point. It is known to extend to some skew linear situations, see $[10,2.5]$. In connection with the Theorem note that not every locally nilpotent irreducible skew linear group is isomorphic to a finitary linear group. Indeed by Section 7 of [13] there exists a locally nilpotent subgroup of a division ring, locally finite-dimensional over its centre, that is not isomorphic to a finitary linear group over any field.

The following proposition concerning locally nilpotent skew linear groups may be of independent interest. Here it arises as a by-product of the proof of the theorem. (Similar conclusions arise in other situations, see 5. below.)

Proposition. Let $G$ be a homogeneous, locally nilpotent subgroup of $G L(n, D)$ and make row $n$-space $V=D^{(n)}$ over $D$ into a $D-G$ bimodule in the obvious way. Then for every element $g$ of $G$ of infinite order we have $V(g-1)=V$ and $C_{V}(g)=\{0\}$.

\section{The proof of the Theorem}

Lemma 1. Let $G$ be a locally nilpotent, completely reducible subgroup of FGL(V) and $X$ a finitely generated subgroup of $G$. Then every $D-X$ composition factor of $V$ embeds into $V$.

This lemma we use as a weak substitute for an extension of the Suprunenko-Tyskevic Theorem. It is also a weak version of the Corollary to the Theorem. 
Proof. By hypothesis $V=\oplus V_{i}$, where the $V_{i}$ are irreducible $D-G$ submodules of $V$. By finitariness only a finite number of the $V_{i}$, say $V_{1}, V_{2}, \ldots, V_{r}$, are non-trivial as $X$-modules. Let $U<W \leqq V$ be $D$ - $X$ submodules of $V$, where $W / U$ is $D-X$ irreducible. Then the $D$ - $X$ series $\{0\} \leqq U<W \leqq V$ and

$$
\{0\}<V_{1}<V_{1} \oplus V_{2}<\cdots<V_{1} \oplus \cdots \oplus V_{r} \leqq V
$$

have isomorphic refinements. Thus, either $W / U$ is isomorphic to some $D-X$ factor of some $V_{i}$ for $1 \leqq i \leqq r$, or, $W / U$ is $X$-trivial, there exists $V_{i}$ for $i$ not in the range $1 \leqq i \leqq r$ and $W / U$ is isomorphic to some $D-X$ submodule of this $V_{i}$. In either case we may assume that $V=V_{i}$; that is, we may assume that $G$ is irreducible.

Since $X$ is finitely generated and finitary $V=V_{X} \oplus C$ as $D-X$ module, where $\operatorname{dim}_{D} V_{X}$ is finite and $C$ is $X$-trivial (see $[11, \S 1]$ ). Let $I$ be a $D-X$ composition factor of $V$. If $I$ is involved in $C$, then $I$ is $D-X$ isomorphic to $D c$ for any $c \neq 0$ in $C$. Hence assume that $I$ is involved in $V_{X}$. Pick $D-X$ submodules $U<W$ of $V_{X}$ with $W / U$ isomorphic to $I$ and $\operatorname{dim}_{D} W$ minimal. We need to prove that $U=\{0\}$, so assume otherwise .

Since $G$ is irreducible $U G=V$. Hence there is a finitely generated subgroup $Y$ of $G$ containing $X$ such that $W \leqq U Y$. By finitariness $\operatorname{dim}_{D} U Y$ is finite. Let $M$ be any maximal $D-Y$ submodule of $U Y$. Since $M \neq U Y$ we have $M \cap U<U$. If $M \cap U<M \cap W$ then since $W / U \cong I$ is $D-X$ irreducible, so $U+(M \cap W)=W$,

$$
W /(M \cap U)=U /(M \cap U) \oplus(M \cap W) /(M \cap U),
$$

$(M \cap W) /(M \cap U) \cong I$ and

$$
\operatorname{dim}_{D}(M \cap W)=\operatorname{dim}_{D} W-\operatorname{dim}_{D}\left(U /(M \cap U)<\operatorname{dim}_{D} W\right.
$$

This contradicts the minimal choice of $W$ and consequently $M \cap U=M \cap W$.

By the Second Isomorphism Theorem $W /(M \cap U)=W /(M \cap W)$ embeds as $D-X$ module into the irreducible finite- $D$-dimensional $D-Y$ module $U Y / M$. Also $X$ is subnormal in $Y$. By Clifford's Theorem $([7,1.1 .7])$ the module $U Y / M$ is completely $D-X$ reducible and therefore so too is $W /(M \cap U)$. Hence there is a $D-X$ submodule $W_{0} \geqq M \cap U$ of $W$ such that $W /(M \cap U)=U /(M \cap U) \oplus W_{0} /(M \cap U)$ and $W_{0} /(M \cap U) \cong$ I. Since $U \neq M \cap U$ this implies that $\operatorname{dim}_{D} W_{0}<\operatorname{dim}_{D} W$ and again we have a contradiction of the minimal choice of $W$. Consequently $U=\{0\}$ and the proof is complete.

For any group $G$ we denote the centre of $G$ by $\zeta_{1}(G)$.

Lemma 2. Let $N$ be an infinite normal subgroup of the finitely generated nilpotent group $G$. Then $N \cap \zeta_{1}(G)$ contains an element of infinite order.

Proof. The group $G$ contains a torsion-free normal subgroup $T$ of finite index. Then $N \cap T$ is non-trivial and normal in $G$. Hence $(N \cap T) \cap \zeta_{1}(G)$ is non-trivial, and necessarily torsion-free. Any of its non-trivial elements has infinite order and lies in $N \cap \zeta_{1}(G)$. 
Lemma 3. Let $N$ be a normal subgroup of finite index in the nilpotent group $G$ and let $Z$ be a finite subset of $\zeta_{1}(N)$. Then $\left\langle Z^{G}\right\rangle \cap \zeta_{1}(G)$ has finite index in $\left\langle Z^{G}\right\rangle$.

Proof. The set $Z^{G}$ is finite (of order at most $|Z| \cdot(G: N)$ ). Thus for some positive integer $m$ the subgroup $A=\left\langle Z^{G}\right\rangle^{m}$ of $\zeta_{1}(N)$ is free abelian of finite rank. But $G$ is nilpotent and $N$ centralizes $A$, so $G / N$ acts on $A$ as a unipotent (and hence torsion-free) group. Therefore $G / C_{G}(A)=\langle 1\rangle$ and so $A \leqq\left\langle Z^{G}\right\rangle \cap \zeta_{1}(G)$. Also $A$ has finite index (at most $\left.m^{|Z| \cdot(G: N)}\right)$ in the abelian group $\left\langle Z^{G}\right\rangle \leqq \zeta_{1}(N)$. The claim follows.

Lemma 4. Let $G$ be a locally nilpotent, homogeneous subgroup of $F G L(V)$ and let $g$ be an element of $G$ of infinite order. Then $[V, g]=V$ and $C_{V}(g)=\{0\}$.

If $G$ in Lemma 4 is merely locally nilpotent and completely reducible then the conclusions of Lemma 4 can be false, even in the linear case with each irreducible constituent non-trivial. For example consider.

$$
G=\langle\operatorname{diag}(2,-1)\rangle \leqq G L(2, \mathbb{Q}) \quad \text { with } \quad g=\operatorname{diag}(4,1)
$$

Proof. Note first that since $g$ is finitary $\operatorname{dim}_{D}[V, g]$ and $\operatorname{dim}_{D}\left(V / C_{V}(g)\right)$ are both finite and equal. Thus $[V, g]=V$ if and only if $C_{V}(g)=\{0\}$. If $W$ is an irreducible $D-G$ submodule of $V$ then $V$ is a direct sum of copies of $W$. If $\left.g\right|_{W}$ has finite order, so does $g$. If $[W, g]=W$ then $[V, g]=V$. Thus we may assume that $V$ is $D-G$ irreducible.

Let $X$ be a finitely generated subgroup of $G$ containing $g$. Suppose $g$ has finite order on each $D-X$ composition factor of $V$. By finitariness $V$ has only a finite number of isomorphism types of $D-X$ composition factors. Hence some positive power of $g$ is unipotent. Since every subgroup of $X$, and in particular $\langle g\rangle$, is subnormal in $X$, it follows that the unipotent radical $u(X)$ of $X$ is not $\langle 1\rangle$, see [9, 2.5]. But by 1.1(b) of [12] the $F$-subalgebra $F[X]$ of End $_{D} V$ generated by $X$ is semiprime and yet $u(X)-1$ generates a nilpotent ideal of $F[X]$ by $[11,2.1 \mathrm{~d}]$. Therefore $g$ has infinite order on some $D-X$ composition factor of $V$. Hence by 1 . above there is some irreducible $D-X$ submodule of $V$ upon which $g$ has inifinite order.

Let $V_{X}$ denote the sum of all the irreducible $D-X$ submodules $W$ of $V$ upon which $g$ has infinite order. Assume that Lemma 4 holds for finitely generated nilpotent groups $G$. Then $[W, g]=W$ for all such $W$ and so $V_{X} \leqq[V, g]$. In particular $V_{X}$ has finite dimension over $D$. Choose a finitely generated subgroup $X$ of $G$ with $g \in X$ and $\operatorname{dim}_{D} V_{X}$ minimal.

Suppose $X$ is normal in some finitely generated subgroup $Y$ of $G$. Let $W$ be an irreducible $D-Y$ submodule of $V$ with $|g|_{w} \mid$ infinite. Let $x \mapsto x^{*}$ denote the restriction map of $Y$ from $V$ to $W$. By Clifford's Theorem $\left(\left[7,1.1 .6\right.\right.$; here $\operatorname{dim}_{D} W$ is finite note $]$ ) we have $W=W_{1} \oplus \cdots \oplus W_{r}$ for the $W_{i}$ the (non-zero) homogeneous components of $W$ as $D-X$ module and $Y$ permutes the $W_{i}$ transitively. Suppose $g$ has finite order on $W_{k}$ for some $k$. Now $N=\cap_{j} N_{Y^{*}}\left(W_{j}\right)$ is a normal subgroup of $Y^{*}$ of finite index. In particular some positive power $h$ of $g^{*}$ lies in $C_{N}\left(W_{k}\right)$. Also $h$ has infinite order. By Lemma 2 there is an element $z$ of $C_{N}\left(W_{k}\right) \cap \zeta_{1}(N)$ of infinite order. Hence by Lemma 3 (with $Z=\{z\}$ ) there is a positive power $z^{m}$ of $z$ central in $Y^{*}$. But $C_{m}\left(z^{m}\right)$ is then a $D-Y$ submodule of 
$W$ containing $W_{k}$ and yet $W_{k} Y=W$. Consequently $z^{m}=1$, a contradiction. For each $k$ this shows that $g$ has infinite order on $W_{k}$ and hence, by homogeneity, on each of its irreducible $D-X$ submodules. Therefore $W \leqq V_{X}$ by definition of $V_{X}$. But then $V_{Y} \leqq V_{X}$, and the minimal choice of $X$ yields that $V_{Y}=V_{X}$.

Now suppose that $Y$ is any finitely generated subgroup of $G$ containing $X$. Then $X$ is subnormal in $Y$. A simple induction on the subnormal depth of $X$ in $Y$ yields that again $V_{Y}=V_{X}$. In particular $V_{X} Y \leqq V_{X}$ for every such $Y$. Consequently $V_{X} G \leqq V_{X}$ and the irreducibility of $G$ yields that $V_{X}=V$. Therefore $V=V_{X} \leqq[V, g] \leqq V$, and the proof will be complete.

We have now reduced the problem to the case where $G$ is irreducible, finitely generated and nilpotent. In particular $\operatorname{dim}_{D} V$ is now finite. We induct on the nilpotency class of $G$. If $G$ is abelian and $x$ is a non-trivial element of $G$ then $[V, x]$ is a non-trivial $D-G$ submodule of $V$ and hence, by irreducibility, it must be $V$. In particular $[V, g]=V$.

Now assume that $G$ is not abelian. Then $H=\left\langle g, G^{\prime}\right\rangle$ is a normal subgroup of $G$ with nilpotency class less than that of $G$. Also $H$ is completely reducible $([7,1.1 .7])$, so suppose $V=V_{1} \oplus \cdots \oplus V_{r}$, where the $V_{i}$ are the non-zero homogeneous components of $V$ as $D-H$ module. By induction we have for each $i$ that either $\left[V_{i}, g\right]=V_{i}$ or $g$ has finite order on $V_{i}$. We require the former to be true for every $i$.

Suppose $g$ has finite order on $V_{j}$ for some $j$ and set $N=\cap_{i} N_{G}\left(V_{i}\right)$. Then $N$ is a normal subgroup of $G$ of finite index and some positive power $h$ of $g$ lies in $C_{N}\left(V_{j}\right)$. Trivially $h$ has infinite order and $N$ is finitely generated and nilpotent. Therefore $C_{N}\left(V_{j}\right) \cap \zeta_{1}(N)$ contains by Lemma 2 an element $z$ of infinite order. Then by Lemma 3 some positive power $z^{m}$ of $z$ lies in $\zeta_{1}(G)$. Hence $C_{V}\left(z^{m}\right)$ is a $D-G$ submodule of the irreducible $D-G$ module $V$ containing $V_{j}$ and yet $V_{j} G=V$. It follows that $z^{m}=1$, a contradiction. This proves that $\left[V_{j}, g\right]=V_{j}$ for each $j$. Consequently $[V, g]=V$, as required.

Clearly the Proposition follows from Lemma 4. It also follows from Lemma 4 that the group $G$ of the Theorem is periodic (and hence locally finite, e.g. [1, 1.A.2]), for if a subgroup $G$ of $F G L(V)$ contains an element $g$ with $[V, g]=V$ (or with $C_{V}(g)=\{0\}$ ) then by finitariness $\operatorname{dim}_{D} V$ is finite. Unlike Lemma 4 above the following partial converse to this remark also applies to periodic groups.

Lemma 5. Let $G$ be a non-trivial locally nilpotent, homogeneous subgroup of $G L(n, D)$. Then $G$ contains an element $g$ with $[V, g]=V$ and $C_{V}(g)=\{0\}$.

Proof. By $[9,8 \mathrm{~b}]$ there is a non-trivial finitely generated subgroup $X$ of $G$, there called a local marker, that is homogeneous. Since $X$ is also nilpotent, the centre of $X$ contains a non-trivial element $g$. Let $W$ be an irreducible $D-X$ submodule of $V$. Since $X$ is homogeneous, $X$ acts faithfully on $W$. Hence $[W, g] \neq\{0\}$ and clearly $[W, g]$ is also a $D-X$ submodule of $W$. Consequently $[W, g]=W$. Again since $X$ is homogeneous we have $[V, g]=V$, and therefore also that $C_{V}(g)=\{0\}$.

Lemma 6. Let $G=P Q$ be an irreducible subgroup of $F G L(V)$, where $P$ and $Q$ are non-trivial subgroups of $G$ satisfying $[P, Q]=\langle 1\rangle$. Then $\operatorname{dim}_{D} V$ is finite. 
Proof. Let $x$ be a non-trivial element of $Q$. Then $[V, x]$ is non-zero, finitedimensional and normalized by $P$. Thus $[V, x] Q=[V, x] G=V$ and so $V$ is generated by finite-dimensional $D$-P submodules, for example the $[V, x] y$ for $y$ in $Q$. In the same way $V$ is generated by finite-dimensional $D-Q$ submodules. Therefore $V=[V, x] Q$ is finite dimensional.

\section{The proof of the Theorem}

As we noted above, Lemma 4 implies that the group $G$ of the theorem is locally finite. Also, being locally nilpotent, $G$ is a direct product of its Sylow subgroups. Thus $G$ is, by Lemma 6 above, a $q$-group for some prime $q$. If $q=\operatorname{char} D$ then $G$ is unipotent ([11, 2.1a]) and hence a stability subgroup ([11, 2.1di]). Thus $G$ is trivial by $[1,2,2 \mathrm{~d}]$ and this contradiction of $\operatorname{dim}_{D} V \geqq 2$ shows that $q \neq \operatorname{char} D$.

By finitariness $V$ is a direct sum of a finite number of irreducible $G$-faithful isomorphic $D-G$ bimodules. Thus we may assume that $V$ is $D-G$ irreducible. Certainly $G \neq\langle 1\rangle$. Pick $x \neq 1$ in $G$ and set $H=\left\langle x^{G}\right\rangle$. Then $H$ is soluble by $[12,1.4]$ (or by [13, $2.2]$ and hence $H$ is completely reducible by $[11,3.1 \& 2.2 \mathrm{~d}]$ and the irreducibility of $G$. Let $V=\oplus_{\omega \in \Omega} V_{\omega}$, where the $V_{\omega}$ are the non-zero homogeneous components of $V$ as $D-H$ module. Then $G$ permutes the $V_{\omega}$, and hence $\Omega$, transitively. In particular if one $V_{\omega}$ is $H$-trivial they all are and hence $V$ is too. Hence no $V_{\omega}$ is $H$-trivial. By $[11,3.2]$ each irreducible $D-H$ submodule of $V$ is finite dimensional. Thus by finitariness $d=\operatorname{dim}_{D} V_{\omega}$ is finite and independent of the choice of $\omega$ in $\Omega$.

Set $N=\cap_{\omega \in \Omega} N_{G}\left(V_{\omega}\right) \geqq H$. Then $S=G / N$ is identified with a locally finite transitive $q$-subgroup of $F \operatorname{Sym}(\Omega)$. Hence by [5, Theorem 2] the set $\Omega$ is countable, as is $S$ itself. In particular, since $\operatorname{dim}_{D} V$ is infinite and $d$ is finite, $|\Omega|=|S|=\operatorname{dim}_{D} V=\aleph_{0}$. Let $W=V_{\omega}$ for some choice of $\omega$ in $\Omega$. Then $\bar{G}=G L(W) l_{\Omega} S$ embeds into $F G L(V)$ via the identification $W=V_{\omega}$ and the action of $S$ on $\Omega$. Let $\pi$ be the natural projection of $G$ onto $S$. Then $g^{-1} \cdot g \pi \in B=\oplus_{\chi \in \Omega} G L\left(V_{\chi}\right)$, the base group of $\bar{G}$, for each $g \in G$, and hence $G$ is a subgroup of $\bar{G}$. Clearly, since $\Omega$ is countable, $G \cap B$ has an ascending series of length $\aleph_{0}$ with factors isomorphic to $q$-subgroups of $G L(W)$. These factors are therefore countable by $[7,2.3 .1$ and 2.5 .2$]$. Also $\bar{G} / B \cong S$ is countable. Therefore $G$ is countably infinite. Finally $G$ is isomorphic by $[13,3.3]$ to a completely reducible monomial finitary linear group over any algebraically closed field of characteristic not $q$.

Lemma 8. Let $G$ be a periodic locally nilpotent subgroup of $F G L(V)$ whose unipotent radical $u(G)$ is trivial. Then every finitely generated subgroup $X$ of $G$ is completely reducible.

Such a group $G$ as in Lemma 8 does have a unipotent radical $u(G)$, for example by $[11,2.1 \mathrm{~d}(\mathrm{i})$ and $2.2(\mathrm{~b})]$. Note that in Lemma 8 the group $G$ need not be completely reducible, even if $G$ is abelian and $\operatorname{dim}_{D} V=2$, see $[7,1.1 .8]$.

Proof. If char $D=p>0$ then $u(G)$ is the set of $p$-elements of $G$ by $[11,2.1(\mathrm{a})]$ and well-known results on nilpotent groups. Thus here either char $D=0$ and $X$ is a finite 
group, or char $D=p>0$ and $X$ is a finite $p^{\prime}$-group. In either case $X$ is completely reducible by 1.1 .3 of [7].

\section{The proof of the Corollary}

If $\operatorname{dim}_{D} V$ is infinite then $G$ is periodic by the Theorem and satisfies $u(G)=\langle 1\rangle$ by [11, $2.2(\mathrm{~d})$ and $2.1 \mathrm{~d}(\mathrm{i})]$. Hence every finitely generated subgroup of $G$ is completely reducible by Lemma 8 above. Now assume that $V$ is finite $D$-dimensional.

Let $X$ be any finitely generated subgroup of $G$. By Lemma 8 of [9] there is a finitely generated subgroup $Y$ of $G$ (namely a "local marker") such that every finitely generated subgroup of $G$ containing $Y$ is completely reducible. In particular $\langle X, Y\rangle$ is completely reducible. But $\langle X, Y\rangle$ is nilpotent, so $X$ is subnormal in $\langle X, Y\rangle$ and hence $X$ is also completely reducible by Clifford's Theorem $([17,1.1 .7])$.

Remark 10. In the proof of Lemma 4 above and hence of the Theorem we have used Part (b) of Theorem 1.1 of [12], but not Part (a). This Part (a) is now an elementary consequence of the Theorem and 7(a) of [9]. Thus if $G$ is a locally nilpotent, homogeneous subgroup of $F G L(V)$ and $F$ is a central subfield of $D$, then the $F$ subalgebra $F[G]$ of End $_{D} V$ generated by $G$ is a crossed product over the local $F C$-centre $\Lambda(G)$ of $G$. The analogues of $[9,7(b) \& 7($ c) $]$ also clearly hold, namely that if $G$ is torsion-free then $F[G]$ is a crossed product over the centre $\zeta_{1}(G)$ of $G$ and if $G / \zeta_{1}(G)$ is finitely generated then $F[G]$ is a crossed product over the $F C$-centre $\Delta(G)$. The first of these follows immediately from the above and the second from [9, 7(c)], since here $V$ is always finite dimensional (even if $G$ is periodic).

Finally in this section we consider possible generalizations of the Corollary to the Theorem.

Lemma 11. Let $N$ be an ascendant subgroup of the subgroup $G$ of $G L(n \cdot D)$. Then the $D-N$ socle of $V=D^{(n)}$ contains the $D-G$ socle of $V$. In particular if $G$ is completely reducible, then so is $N$.

Proof. Suppose $N=N_{0} \unlhd N_{1} \unlhd \cdots \unlhd N_{\alpha} \unlhd \cdots \unlhd N_{\gamma}=G$. Let $S_{\alpha}$ denote the $D-N_{\alpha}$ socle of $V$. We prove by induction on $\gamma$ that $S_{0} \geqq S_{\gamma}$. If $\gamma-1$ exists then $S_{0} \geqq S_{\gamma-1}$ by induction. If $W$ is an irreducible $D-G$ submodule of $V$ then $W$ is $D-N_{\gamma-1}$ completely reducible by Clifford's Theorem $([7,1.1 .7])$ and so $W \leqq S_{\gamma-1}$. Therefore $S_{\gamma} \leqq S_{y-1} \leqq S_{0}$.

Now suppose $\gamma$ is a limit ordinal. By induction $S_{\alpha} \geqq S_{\beta}$ whenever $0 \leqq \alpha \leqq \beta<\gamma$. Choose $\alpha<\gamma$ such that $\operatorname{dim}_{D} S_{\alpha}$ is minimal. Then $S_{\alpha}=\mathbf{S}_{\beta}$ for all $\alpha \leqq \beta<\gamma$ and so $S_{\alpha}$ is a $\bigcup_{\alpha \leq \beta<\gamma} N_{\beta}=G$ submodule of $V$. Clearly $W \cap S_{\alpha} \neq\{0\}$ for every irreducible $D-G$ submodule $W$ of $V$. Consequently $W \leqq S_{\alpha}$ and so $S_{\alpha} \geqq S_{\gamma}$. Since $S_{0} \geqq S_{\alpha}$ the result follows.

Corollary 12. Let $G$ be a locally nilpotent, completely reducible skew linear group.

(a) If $G$ is a hypercentral group (more generally if $G$ is an $N$-group in the sense of Kuros, see [6, page 2], then every subgroup of $G$ is completely reducible. 
(b) If $G$ is periodic then every subgroup of $G$ is completely reducible.

(c) If $X$ is a subgroup of $G$ such that $X$ modulo the hypercentre $\zeta(G)$ of $G$ is finitely generated, then $X$ is completely reducible.

(d) If $X$ is a subgroup of $G$ such that $X \cdot \Lambda(G) / \Lambda(G)$ is finitely generated, then $X$ is completely reducible.

(Again $\Lambda(G)$ denotes the local $F C$-centre of $G$ ).

Proof. (a) Here every subgroup of $G$ is ascendant in $G$, so (a) follows at once from Lemma 11 above.

(b) Each Sylow subgroup of $G$ is a Cernikov group by $[7,2.3 .1,2.5 .2,1.3 .3]$ and the linear case. Thus $G$ has an ascending characteristic series with finite factors. Being locally nilpotent, it follows that $G$ is a hypercentral group and hence Part (a) applies.

(c) We modify the proof of [9, Point 8a]. Choose a subgroup $X$ of $G$ with $X \cdot \zeta(G) / \zeta(G)$ finitely generated, such that the composition length of $D^{(n)}$ as $D-X$ module is minimal and, given that, such that the $D$-dimension of the $D-X$ socle of $V$ is also minimal. If $Y$ is any subgroup of $G$ containing $X$ with $Y \cdot \zeta(G) / \zeta(G)$ finitely generated, the argument of [9, Point 8a] shows that $Y$ is completely reducible. Also $Y \cdot \zeta(G) / \zeta(G)$ is nilpotent, so $Y$ is hypercentral and hence by (a) every subgroup of $Y$ is completely reducible. Every subgroup of $G$ that is finitely generated modulo $\zeta(G)$ is a subgroup of some such $Y$ and hence is also completely reducible.

(d) The maximal torsion subgroup $T$ of $\Lambda(G)$ has, as in (b), an ascending characteristic series with finite factors, so $T \leqq \zeta(G)$. Also $\Lambda(G) / T$ is the centre of $G / T$, see [9, Point 6], and hence $\Lambda(G) \leqq \zeta(G)$. Therefore (d) follows from (c).

\section{The examples}

Lemma 13. Let $D$ be a division ring, $W$ a finite-dimensional left $D$-space, $H a$ non-trivial irreducible subgroup of $G L(W), \Omega$ a non-empty set and $K$ a transitive subgroup of $F \operatorname{Sym}(\Omega)$. Then $H l_{\Omega} K$ is isomorphic to an irreducible subgroup of $F G L(V)$ for $V=\bigoplus_{\omega \in \Omega} W_{\omega}$ a direct sum of $|\Omega|$ copies $W_{\omega}$ of $W$.

Proof. Choose for each $\omega$ in $\Omega$ a $D$-isomorphism of $W$ to $W_{\omega}$. This determines a subgroup $H_{\omega}$ of $F G L(V)$ isomorphic to $H$, that acts on $W_{\omega}$ via this isomorphism as $H$ acts on $W$ and acts as $\langle 1\rangle$ on the $W_{\sigma}$ for all $\sigma \neq \omega$. Then $B=\left\langle H_{\omega}: \omega \in \Omega\right\rangle \leqq F G L(V)$ is the direct product of the $H_{\omega}$. Also $G L(V)$ contains a subgroup $S$ isomorphic to $K$ that acts by permuting the $W_{\omega}$ via the action of $K$ on $\Omega$. Since $\operatorname{dim}_{D} W$ is finite and $K$ is finitary by hypothesis, we have $S \leqq F G L(V)$ and so $F G L(V)$ contains the subgroup $G=S B \cong H l_{\Omega} K$.

It remains only to prove that $G$ is irreducible. Let $U$ be non-zero $D-G$ submodule of $V$. Since $H$ is non-trivial and irreducible, the $W_{\omega}$ are the homogeneous components of $V$ as $D-B$ module and each $W_{\Omega}$ is $D-B$ irreducible. Hence there is some $\omega$ in $\Omega$ with $W_{\omega} \leqq U$ and then $U \geqq U S \geqq W_{\omega} S=V$, using, finally, that $K$ is transitive. Therefore $G$ is irreducible. 
Remark 14. Assuming $K$ as in Lemma 13 is non-trivial, the subgroup $S$ of $F G L(V)$ is never irreducible, for with $S$ acting trivially on $W$, the linear map of $V$ onto $W$ extending the chosen isomorphisms of the $W_{\omega}$ to $W$ is an $S$-map and its kernel is a proper $D-S$ submodule of $V$. If $K$ is locally soluble (e.g. if $K$ is locally nilpotent), then $|\Omega| \leqq \aleph_{0}$ by Theorem 2 of $[5]$ and $\operatorname{dim}_{D} V \leqq \aleph_{0}$.

Example 15. Let $q$ be a prime, $F$ a field with a primitive $q$ th root $\zeta$ of unity, $\Omega$ a countable set and $K$ an infinite transitive $q$-subgroup of $F \operatorname{Sym}(\Omega)$. Then the group $G=\langle\zeta\rangle l_{\Omega} K$ is isomorphic to a locally nilpotent infinite-dimensional finitary skew linear group.

For $G$ is a locally finite $q$-group and hence is locally nilpotent and $G$ is isomorphic to an irreducible subgroup of $F G L\left(F^{(\Omega)}\right)$ by Lemma 13. An example of the group $K$ as in Example 15 would be the wreath power of the cyclic group of order $q$ in its regular representation over the set of positive integers, see [6, pp. 18-22].

Example 16. Let $p$ be zero or a prime and $n$ and $c$ positive integers. Then there exists a torsion-free nilpotent irreducible skew linear group of characteristic $p$, degree $n$ and class $c$.

Proof. Suppose $E=F(h)$ is a finite field extension of the field $F$ of degree $n$ such that $h$ is not a root of unity. Let $K$ be any torsion-free nilpotent group of class $c$ and set $D=F(K)$, the division ring of quotients of the group algebra $F K$. Choose an $F$-basis of $E$. This identifies $h$ with an element of $G L(n, F)$. Set $G=\langle h, K\rangle \leqq G L(n, D)$. Then $G=\langle h\rangle \times K$ is torsion-free and nilpotent of class $c$.

We have to prove that $G$ is irreducible. Let $U$ be a $D-G$ composition factor of row $n$ space $V=D^{(n)}$ over $D$, say of dimension $m \leqq n$. Since $E=F[h]$ is a field, $E$ acts faithfully on $U$. Hence there is an $F$-embedding of $E$ into $D^{m \times m}$. By [3, Point 2 (final part)] we have that $n=\operatorname{dim}_{F} E \leqq m$. Therefore $m=n, U=V$ and $G$ is irreducible.

We should finish this construction by pointing out that there are many examples of $F, E$ and $h$ as above. For example let $F=\mathbb{Q}$, let $h$ be a root of say $X^{n}-2$ and $E=F(h)$. Then $(E: F)=n$ by Eisenstein's Criterion and clearly $|h|$ is infinite. This settles the case $p=0$. Suppose $p>0$. Let $k$ be the prime field of characteristic $p$ and $x$ an indeterminate over $k$. Then $k(x)=\bigoplus_{0 \leqq i<n} x^{i} \cdot k\left(x^{n}\right)$ and so $\left(k(x): k\left(x^{n}\right)\right)=n$. Set $E=k(x), F=k\left(x^{n}\right)$ and $h=x$. Trivially $h$ has infinite order.

Let $G$ be a subgroup of $F G L(V)$. We call a $D-G$ submodule $U$ of $V$ primary if the $D-G$ composition factors of $U$ are all isomorphic. Suppose $G$ is locally nilpotent and $V$ is finite dimensional. It is an old result (1959) of D. A. Suprunenko \& R. Tyskevic (e.g. [8, 7.15]) that if $D$ is an algebraically closed field then $V$ is a direct sum of primary $D-G$ submodules. This generalizes considerably-the same conclusion holds if $D$ is any finitedimensional division algebra, see $[10,2.5]$. If $V$ is a direct sum of primary $D-G$ submodules clearly every $D-G$ composition factor of $V$ embeds into $V$ (we are still assuming $\operatorname{dim}_{D} V$ is finite). Thus if the conclusion held for arbitrary division rings $D$ 
then Lemma 1 above would be an immediate corollary. Moreover in view of $[12$, Theorem $1 \mathrm{~b}$ ] we would only need this conclusion for groups $G$ such that the subalgebra of End ${ }_{D} V$ generated by $G$ over any central subfield of $D$ is semiprime. However, even in these special circumstances, the conclusion is false. Before we give an example we need to present the following technical lemma.

Lemma 17. Let $F$ be a field, $E$ a division $F$-algebra, $\left\{a_{i}: i \in \mathbb{Z}\right\}$ a set of commuting independent indeterminates over $E$ and $L=E\left(a_{i}: i \in \mathbb{Z}\right)$ the corresponding division ring of quotients. Let $g$ be the $F$-automorphism of $L$ satisfying $\left.g\right|_{E}=1$ and $a_{i}^{g}=a_{i+1}$ for each $i$. Suppose $a_{i} \delta=\delta^{g}+\eta$ for some $i \in \mathbb{Z}, \delta \in L$ and $\eta \in E$. Then $\delta=\eta=0$.

Proof. If $E$ is a field each element $\delta$ of $L \backslash\langle 0\rangle$ has a well-defined support, namely the set of integers $i$ for which $a_{i}$ is involved in $\delta$, and then a simple argument proves Lemma 17. If $E$ is not commutative it seems we need more care.

Order the free abelian group $A=\left\langle a_{i} ; i \in \mathbb{Z}\right\rangle$ lexicographically using $a_{i}<a_{i+1}$ for each $i$; specifically, in vector notation set $\underline{a}^{e}<\underline{a}^{f}$ if for some $i$ we have $e_{i}<f_{i}$ but $e_{j}=f_{j}$ for all $j<i$. With this ordering let $L^{*}=E((A))=E\left(\left(a_{i}: i \in \mathbb{Z}\right)\right)$ be the division ring of formal sums over well-ordered subsets of $A$ with coefficients in $E$, cf. $[7,1.4 .14 \&$ proof]. Note that $L$ embeds into $L^{*}$ via the obvious embedding of $E A$ in $L^{*}$. Now each element of $L^{*}$ has a unique expression as a formal sum (possibly over the empty set) and hence we can define a support $S(\lambda)$ for $\lambda$ in $L^{*}$ by $j \in S(\lambda)$ if and only if there is a monomial in the $a_{i}$ with the exponent of $a_{j}$ non-zero for which the coefficient (in $E$ ) in the formal sum expression for $\lambda$ is non-zero. (In general $S(\lambda)$ could be infinite, but certainly it is finite whenever $\lambda$ lies in $L$. Also $S(\eta)=\varnothing$ for all $\eta \in E$.)

It is easy to see that the action of $g$ on $A$ preserves the order. Thus the action of $g$ on $L$ extends to one on $L^{*}$ such that $S(\lambda g)=\{j+1: j \in S(\lambda)\}$ for any $\lambda$ in $L^{*}$. We are given $a_{i} \delta=\delta^{g}+\eta$ for some $\delta \in L$ and $\eta \in E$. Then $\{i\} \cup S(\delta)=\{i\} \cup S\left(\delta^{g}\right)$. Also $S(\delta)$ is finite; assuming $S(\delta) \neq \varnothing$ let $r$ be its least member and $s$ its greatest member. Then $r \notin S\left(\delta^{\theta}\right)$, so $r=i$, and $s+1 \in S\left(\delta^{g}\right) \backslash S(\delta)$, so $s+1=i$. But $r \leqq s<s+1$. This contradiction shows that $S(\delta)=\varnothing$ and so $\delta \in E$. If $\delta \neq 0$ then $\{i\}=S\left(a_{i} \delta\right)=S\left(\delta^{g}\right)=\varnothing$, which is also impossible. Consequently $\delta=0=\delta^{g}$ and $\eta=0$.

Lemma 18. Assume the notation of Lemma 17 and let $D=L((g))$ be the division ring of skew Laurent series in $g$ over L. Let

$$
a=\left(\begin{array}{cc}
g & 0 \\
\gamma & g a_{0}
\end{array}\right) \in G L(2, D)
$$

for some $\gamma \in E \backslash\{0\}$. Set $A=\langle a\rangle \leqq G L(2, D)$ and $V=D^{(2)}$, regarded as a $D-A$ bimodule in the obvious way. Put $U=D e_{1}$ for $e_{1}=(1,0)$. Then the following hold.

(a) $F[A] \leqq D^{2 \times 2}$ is an integral domain.

(b) $U$ is a $D-A$ submodule of $V$ and $V$ does not split over $U$.

(c) $U$ and $V / U$ are non-isomorphic irreducible $D-A$ modules.

(d) $V$ is not completely reducible as $D-A$ module. 
(e) $V$ is not a direct sum of primary $D-A$ modules.

Proof. (a) $F[A]$ is isomorphic to the group algebra of the infinite cyclic group over $F$ and hence is an integral domain.

(b) Trivially $U A \leqq U$. Suppose $V$ splits over $U$. Then $V=U \oplus D w$ as $D-A$ module for some $w$ and we can choose $w=(d, 1)$ for some $d$ in $D$. Also $w a=d^{\prime} w$ for some $d^{\prime} \in D$. Hence $d g+\gamma=d^{\prime} d$ and $g a_{0}=d^{\prime}$, and hence $d g+\gamma=\mathrm{ga}_{0} d$. Suppose $d=\sum_{i \geq r} g^{i} \delta_{i}$, where the $\delta_{i}$ lie in $L$. Then

$$
\gamma+\sum g^{i+1} \delta_{i}^{g}=\sum g^{i+1} a_{i} \delta_{i}
$$

and so $\delta_{-1, i} \gamma+\delta_{i}^{g}=a_{i} \delta_{i}$ for each $i$. By Lemma 17 the $\delta_{i}$ and $\gamma=\delta_{-1,-1} \gamma$ are zero. This contradiction of the assumption that $\gamma \neq 0$ shows that $V$ does not split over $U$.

(c) Suppose $\phi$ is a $D-A$ isomorphism of $V / U$ to $U$ and let $\left(e_{2}+U\right) \phi=\mathrm{de}_{1}$ for some $d$ in $D$, where $e_{2}=(0,1)$. Then

$$
d g e_{1}=\left(d e_{1}\right) a=\left(e_{2}+U\right) \phi a=\left(e_{2}+U\right) a \phi=\left(g a_{0} e_{2}+U\right) \phi=g a_{0}\left(\left(e_{2}+U\right) \phi\right)=\operatorname{ga}_{0} \mathrm{de}_{1} .
$$

Hence $d g=g a_{0} d$. Again let $d=\sum_{i \geqq r} g^{i} \delta_{i}$ for $\delta_{i} \in L$. Then

$$
\sum g^{i+1} \delta_{i}^{g}=\sum g^{i+1} a_{i} \delta_{i}
$$

and so $\delta_{i}^{g}=a_{i} \delta_{i}$ for each $i$. By Lemma 17 each $\delta_{i}=0$ and so $d=0$. This is impossible if $\phi$ is an isomorphism, so $U$ and $V / U$ are not $D-A$ isomorphic.

(d) This follows at once from (b).

(e) This follows at once from (c) and (b).

Remark 19. In Lemma 18, instead of taking $L((g))$ for $D$, we could take the division ring $L(g)$ of quotients of the skew polynomial ring $L[g]$, since the latter is isomorphic to a subring of the former in a natural way.

The group $A$ in Lemma 18 is abelian. There exist comparable examples that are nilpotent of arbitrary (positive) nilpotency class. More generally we have the following.

Example 20. Let $G$ be any torsion-free locally soluble group with an infinite cycle image and let $F$ be any field. Then there exists a division $F$-algebra $D$ and a $G$-faithful $D-G$ bimodule $V$ of $D$-dimension 2 with a $D-G$ submodule $U$, such that the following hold.

(a) The $F$-subalgebra of $\operatorname{End}_{D} V$ generated by the image of $G$ is just the group algebra $F G$. In particular it is an Ore domain. Also the subalgebra of $\operatorname{End}_{D} V$ generated by the image of $G$ over any central subfield $Z$ of $D$ is a domain.

(b) $V$ does not split as $D-G$ module over $U$.

(c) $U$ and $V / U$ are non-isomorphic irreducible $D-G$ modules.

(d) $V$ is not completely reducible as $D-G$ module.

(e) $V$ is not a direct sum of primary $D-G$ modules. 
Note that if $G$ is any non-trivial torsion-free nilpotent group then $G$ does have an infinite cycle image.

Proof. Let $\left\{b_{i}: i \in \mathbb{Z}\right\}$ be a set of independent commuting indeterminates over $F$ and set $K=F\left(b_{i}: i \in \mathbb{Z}\right)$. By hypothesis $G$ is the split extension by a normal subgroup $N$ by an infinite cyclic group $H=\langle h\rangle$. Let $G$ act on $K$ as a group of $F$-automorphisms, where $N$ centralizes $K$ and $b_{i}^{h}=b_{i+1}$ for each $i$. The resulting skew group algebra $K G$ has (see [2]) a division ring $D$ of quotients. Then $E=F(N)$ is a division subring of $D$, the $b_{i}$ are independent over $E$ commuting with $E$ and $D=L(h)$ for $L=E\left(b_{i}: i \in \mathbb{Z}\right)$. We are in the situation of Lemma 17 above, but with $b_{i}$ and $h$ in place of $a_{i}$ and $g$. We set $a_{i}=b_{i}^{-1}$ and $g=h b_{0}$.

Define an $F$-automorphism $\sigma$ of $D$ by $l^{\sigma}=h \mid l h^{-1}$ for $l \in L$ and $h^{\sigma}=h b_{0}$. Since $\left.h\right|_{L}=\left.h b_{0}\right|_{L}$ certainly this defines an automorphism of the skew group ring $L\langle h\rangle$ and $D$ is the ring of quotients of $L\langle h\rangle$. Thus $\sigma$ is a well-defined automorphism of $D$.

We now define an $F$-linear map $\delta$ of $D$ into itself such that $L \delta=\{0\}, h \delta=1$ and (de) $\delta=d \delta \cdot e^{\sigma}+d \cdot e \delta$ for all $d, e \in D$. (The latter says that $\delta$ is a $\sigma$-derivation.) Let $D={ }_{1} D_{\sigma}$ denote the $D-D$ bimodule given by $d \cdot x \cdot e=d x e^{\sigma}$ for all $d, e, x \in D$. Let $A=D \oplus_{1} D_{a}$ be the $F$-algebra whose multiplication is given by

$$
(d, x)(e, y)=\left(d e, x e^{\sigma}+d y\right)
$$

for all $d, e \in D$ and $x, y \in{ }_{1} D_{\sigma}$ and whose $F$-space structure is given by that of $D$ and ${ }_{1} D_{\sigma}$. Note that if $d \neq 0$ then $(d, x)$ is a unit of $A$ with inverse given by

$$
(d, x)^{-1}=\left(d^{-1},-d^{-1} x d^{-\sigma}\right)
$$

Define an $F$-algebra homomorphism $\varepsilon$ of $D$ into $A$ by $l \varepsilon=(l, 0)$ for $l \in L$ and $h \varepsilon=(h, 1)$ as follows. Now $(h \varepsilon)^{-1}=\left(h^{-1},-h^{-1} h^{-\sigma}\right)$ and for all $l$ in $L$

$$
\begin{aligned}
(h \varepsilon)^{-1}(l \varepsilon)(h \varepsilon) & =\left(h^{-1},-h^{-1} h^{-\sigma}\right)(l h, l) \\
& =\left(l^{h}, h^{-1} l-h^{-1} h^{-\sigma} l h^{\sigma}\right) \\
& =\left(l^{h}, h^{-1} l-h^{-1}\left(l^{h}\right)^{\sigma}\right)=l^{h} \varepsilon
\end{aligned}
$$

since $\left.\sigma\right|_{L}=\left.h^{-1}\right|_{L}$. Thus $\varepsilon$ defines a ring injection of $L\langle h\rangle$ into $A$. If $\pi: A \rightarrow D$ is the projection of $A$ onto the first coordinate then $\varepsilon \pi$ is one-to-one on $L\langle h\rangle$. Thus $L\langle h\rangle \varepsilon \backslash\{0\}$ consists of units of $A$ and so $\varepsilon$ extends uniquely to an $F$-algebra homomorphism of $D$ into $A$. Define $\delta: D \rightarrow D$ by $d \varepsilon=(d, d \delta)$. By definition $L \delta=\{0\}$ and $h \delta=1$. Also for $d$ and $e$ in $D$ we have

$$
(d e,(d e) \delta)=(d e) \varepsilon=(d \varepsilon)(e \varepsilon)=\left(d e,(d \delta) e^{\sigma}+d(e \delta)\right)
$$

The construction of $\delta$ is complete.

Now consider the skew polynomial ring $S=D[x ; \sigma, \delta]$, where the multiplication is 
given by $d x=x d^{\sigma}+d \delta$ for all $d \in D$, cf. [4, p. 15]. Then $S$ is a Noetherian domain ([4, 2.9]) and so $S$ has a division ring $D_{0}$ of quotients.

Let $G^{*}$ denote the subgroup of $G L(2, D)$ given by

$$
\left(\begin{array}{cc}
h b_{0} & 0 \\
1 & h
\end{array}\right) \cdot\left(\begin{array}{cc}
n & 0 \\
0 & n^{h}
\end{array}\right): n \in N
$$

Note that in $G L\left(2, D_{0}\right)$ we have

$$
\left(\begin{array}{ll}
1 & 0 \\
x & 1
\end{array}\right)\left(\begin{array}{cc}
h b_{0} & 0 \\
1 & h
\end{array}\right)\left(\begin{array}{cc}
1 & 0 \\
-x & 1
\end{array}\right)=\left(\begin{array}{cc}
h b_{0} & 0 \\
x h b_{0}+1-h x & h
\end{array}\right)
$$

and $x h b_{0}+1-h x=x h b_{0}+1-\left(x h^{\sigma}+h \delta\right)=0$, and

$$
\left(\begin{array}{ll}
1 & 0 \\
x & 1
\end{array}\right)\left(\begin{array}{ll}
n & 0 \\
0 & n^{h}
\end{array}\right)\left(\begin{array}{cc}
1 & 0 \\
-x & 1
\end{array}\right)=\left(\begin{array}{cc}
n & 0 \\
x n-n^{h} x & n^{h}
\end{array}\right)
$$

and $x n-n^{h} x=x n-\left(x n^{h \sigma}+n^{h} \delta\right)=0$. Thus in $G L\left(2, D_{0}\right)$ the group $G^{*}$ is conjugate to

$$
\left\langle\operatorname{diag}\left(h b_{0}, h\right), \operatorname{diag}\left(n, n^{h}\right): n \in N\right\rangle,
$$

which is isomorphic to $G$ via $h^{i} n \mapsto \operatorname{diag}\left(\left(h b_{0}\right)^{i} n, h^{i} n^{h}\right)$. In particular this and the above conjugation determine an $F$-algebra isomorphism of $F G$ onto $F\left[G^{*}\right] \leqq D^{2 \times 2}$. Hence $F\left[G^{*}\right]$ is an Ore domain. The central subfield $Z$ of $D$ lies in $L$, for it is easy to check that the centralizer of $b_{0}$ in $L((h)) \geqq D$ lies in $L$. Hence $\left.\sigma\right|_{Z}$ is the identity on $Z,\left.\delta\right|_{Z}$ is the zero on $Z$ and $x$ commutes with $Z$. Consequently the same isomorphism of $G$ yields an isomorphism of $Z\left[G^{*}\right] \leqq D^{2 \times 2}$ with the subdomain $Z[G]$ of $D$. (One only needs to check this on $Z[N]$.) This yields $a$ ) with $V=D^{(2)}$ of course.

Let

$$
a=\left(\begin{array}{cc}
g & 0 \\
1 & g a_{0}
\end{array}\right) \in G^{*}
$$

where $g=h b_{0}$ and $a_{i}=b_{i}^{-1}$ for each $i$. If $V$ splits as $D$-G* module over $U=D e_{1}$ for $e_{1}=(1,0)$, then $a$ has an eigenvector in $V \backslash U$ over $D$ and hence also over the larger ring $L(g)) \geqq L(g)=L(h)=D$. This contradicts Lemma $18(\mathrm{~b})$. Then $V$ does not split over $U$, which yields (b). By 18 (c) also $U$ and $V / U$ are not isomorphic as $D-\langle a\rangle$ module, for otherwise $L((g)) U$ and $L((g)) V / L((g)) U$ would be $L(g))-\langle a\rangle$ isomorphic. Hence they cannot be isomorphic as $D-G^{*}$ module either. This yields (c), and (d) and (e) follow from (b) and (c).

Remark 21. The groups $A$ of Lemma 18 and $G^{*}$ of Example 20 do not lie in any 
locally nilpotent completely reducible finitary skew linear group over $D$, for if this were the case the same would apply to the finitely generated subgroups of $G^{*}$ (the group $A$ is already finitely generated). Now Example 20 applies equally well to any finitely generated subgroup of $G$ containing $h$. Thus $18(d)$ and $20(d)$ would yield a contradiction to the Corollary to the Theorem.

\section{REFERENCES}

1. O. H. Kegel, and B. A. F. Wehrfritz, Locally Finite Groups (North-Holland Pub. Co., Amsterdam, 1973).

2. P. H. Kropholler, P. A. Linnell and J. A. Moody, Applications of a new K-theoretic theorem to soluble group rings, Proc. Amer. Math. Soc. 104 (1988), 675-684.

3. A. I. Lichtman and B. A. F. Wehrfritz, Finite dimensional subalgebras in matrix rings over transcendental division algebras, Proc. Amer. Math. Soc. 106 (1989), 335-344.

4. J. C. McConnell and J. C. Robson, Non-commutative Noetherian Rings (John Wiley \& Sons, Chichester, 1987).

5. P. M. Neumann, The lawlessness of groups of finitary permutations, Arch. Math. 26 (1975), 561-566.

6. D. J. S. Robinson, Finiteness Conditions and Generalized Soluble Groups Vol 2 (SpringerVerlag, Berlin, 1972).

7. M. Shirvanı and B. A. F. Wehrfritz, Skew Linear Groups (Cambridge Univ. Press, Cambridge, 1986).

8. B. A. F. Wehrfritz, Infinite Linear Groups (Springer-Verlag, Berlin, 1973).

9. B. A. F. Wehrfritz, Soluble and locally soluble skew linear groups, Arch. Math. 49 (1987), 379-388.

10. B. A. F. Wehrfritz, Some nilpotent and locally nilpotent matrix groups, J. Pure Appl. Algebra 60 (1989), 289-312.

11. B. A. F. Wehrfritz, Locally soluble finitary skew linear groups, J. Algebra 60 (1993) 226-241.

12. B. A. F. Wehrfritz, Algebras generated by locally nilpotent finitary skew linear groups, $J$. Pure App. Algebra 88 (1993), 305-316.

13. B. A. F. Wehrfritz, Locally nilpotent finitary skew linear groups, J. London Math. Soc., to appear.

School of Mathematical Sciences

Queen Mary and Westerfield College

Mile ENd RoAd

LONDON E1 4NS

ENGLAND 